\title{
Não-fechamento dos Peritônios Visceral e Parietal na Operação Cesariana
}

\author{
Nonclosure of the Visceral and Parietal Peritoneum at Cesarean Section \\ Olimpio Barbosa de Moraes Filho, Cícero Ferreira Fernandes Costa
}

\begin{abstract}
RESUM0
Objetivo: determinar se o não-fechamento dos folhetos peritoneais (visceral e parietal) na cesárea apresenta beneficios no intra e pós-operatório.

Pacientes e Métodos: seiscentas e noventa e oito mulheres programadas para cesárea foram alocadas aleatoriamente em dois grupos: com sutura dos peritônios visceral e parietal $(n=$ 349) e sem sutura dos peritônios ( $n=349$ ), na Maternidade da Encruzilhada (CISAM) em Recife, entre novembro de 1997 e dezembro de 1998. A análise estatística comparou as variáveis do intra-operatório e do pós-operatório entre os dois grupos. Não houve diferenças significativas entre os dois grupos em relação a idade, paridade, idade gestacional, antibiótico profilático, cefaléia pós-raquianestesia, cistite, amniorrexe prematura e indicações da cesárea. Resultados: o tempo cirúrgico, o número de fios categute simples e o uso de analgésico foram significativamente menores no grupo sem sutura do que no grupo com sutura. As incidências de febre, infecção de ferida operatória, endometrite foram similares nos dois grupos. Não houve diferenças quanto ao uso de antifisético, antiemético e óleo mineral. As médias de dias de permanência hospitalar foram similares nos dois grupos.

Conclusões: o não-fechamento dos folhetos peritoneais não apresenta efeitos adversos no pós-operatório e, ao contrário, diminui o uso de analgésicos e no intra-operatório diminui o tempo e o número de fios categute simples.
\end{abstract}

PALAVRAS-CHAVE: Cesárea. Cirurgia: complicações. Infecção puerperal.

\section{Introdução}

A sutura dos peritônios visceral e parietal na cesárea tem sido largamente aceita, apesar da inexistência de estudos que demonstrem seu beneficio. Também, não se sabe ao certo quando a sutura do peritônio passou a fazer parte da prática cirúrgica.

Uma justificativa empírica para a realização da sutura do peritônio seria impedir o ingresso de sangue e de tecido rico em fibrina na cavidade peritoneal durante o pós-operatório imediato, teoricamente diminuindo a chance de formação de aderências ${ }^{1,2}$. O fechamento peritoneal passou a ser rotineiramente realizado sem haver, na verdade, nenhuma comprovação científica de suas vantagens.

Departamento Materno-Infantil da Faculdade de Ciências Médicas da Universidade de Pernambuco, Recife, PE

Correspondência:

Olimpio Barbosa de Moraes Filho

Rua Quarenta e oito, n ${ }^{\circ} 97$ / 101 - Espinheiro

52020-060 - Recife - PE
Alguns pesquisadores, realizando estudos em ratos, encontraram uma menor incidência de aderência quando o peritôneo não era suturado $^{3,4,5,6,7}$. Outro estudo realizado em coelhas mostrou que a cicatrização do peritônio parietal ocorre entre 48 e 72 horas quando não suturado e que a reaproximação das bordas resulta no aumento de necrose, inflamação e reação de corpo estranho ao fio, retardando o processo de cura ${ }^{8}$. Também em animais foi observado que, tanto com peritonite ou não, é menor a incidência de aderências após laparotomias, quando não suturado o folheto peritoneal ${ }^{9}$.

Estudos controlados em seres humanos laparotomizados mostraram que não há diferença significativa em relação à cura da ferida, quando se compara um grupo com sutura e outro grupo sem sutura do peritônio ${ }^{10,11}$. Em 1987, estudo prospectivo randomizado em 206 pacientes submetidos à laparotomia por incisão paramediana lateral concluiu que não se faz necessário fechamento do peritônio parietal ${ }^{12}$.

Em Quebec, Canadá, 483 mulheres estéreis, 
submetidas a anastomoses tubárias, salpingostomias ou fimbrioplastias, foram separadas em dois grupos: o primeiro grupo com fechamento do folheto peritoneal e no segundo o peritônio foi deixado aberto. Após seis meses, foram realizadas laparoscopias naquelas que não tinham engravidado, não sendo observado diferença entre a incidência de aderências entre os grupos ${ }^{13}$.

As vantagens e deméritos do fechamento peritoneal, no momento da cesárea, foram também avaliados em 248 pacientes separadas em dois grupos: com o peritônio fechado da maneira habitual ou abandonado sem qualquer sutura ${ }^{14}$. O tempo médio de cirurgia no grupo sem fechamento foi menor, mas não houve diferenças no pós-operatório quanto à incidência de infecções da ferida, de deiscências, endometrite, íleo e duração da permanência hospitalar. O estudo sugere que deixar o peritônio parietal sem sutura é procedimento recomendável na realização da cesárea ${ }^{14}$.

Hull e Varner ${ }^{15}$ chegaram à conclusão que o não-fechamento dos peritônios visceral e parietal, na operação cesariana, não apresenta efeitos adversos na recuperação pós-operatória, diminui o uso de narcóticos, permite o retorno mais rápido das funções intestinais e simplifica e encurta o procedimento cirúrgico.

Em Israel foram efetuadas 467 cesáreas, sendo as pacientes divididas em dois grupos: um com incisão de Pfannenstiel e fechamento de ambos os folhetos peritoneais e outro com método de Joel-Cohen para abertura do ventre, deixando os peritônios parietal e visceral abertos. A incidência de febre no pós-operatório foi menor no grupo que empregou o método de Joel-Cohen ${ }^{16}$.

Em outro estudo realizado em Israel, 200 mulheres submetidas a cesareana foram prospectivamente randomizadas, o primeiro grupo com sutura do peritônio visceral e parietal e o segundo, sem sutura dos peritônios. A técnica sem peritonização levou à redução do tempo de cirurgia, sem apresentar efeitos adversos no pós-operatório ${ }^{17}$.

Em outro estudo comparativo com 546 cesáreas (262 sem sutura e 287 com sutura), foi observado que a duração da anestesia e a freqüência de intercorrências febris no pós-operatório, com maior necessidade de analgesia e de antibióticos, foram mais elevadas quando o peritônio havia sido fechado ${ }^{18}$.

Estudo realizado no Canadá e na Suiça comparou 137 cesáreas em que não se suturaram os folhetos peritoneais com outras 143 nas quais os folhetos foram fechados com categute. A média do tempo de cirurgia foi mais curta em seis minutos quando se fez a sutura ${ }^{19}$.

Assim como a revisão exaustiva sobre o as- sunto realizado por Wadstrom e Gerdin ${ }^{20}$, também não encontramos estudos que mostrem alguma vantagem na peritonização.

Este estudo objetiva comparar os dados maternos de pacientes submetidas à operação cesariana sem a peritonização dos folhetos visceral e parietal com igual número de pacientes submetidas a cesareana cuja peritonização foi realizada.

\section{Pacientes e Métodos}

Entre novembro de 1997 e dezembro de 1998, 698 parturientes que foram submetidas a operação cesariana foram alocadas por seqüência em dois grupos: com sutura dos folhetos peritoneais ( $\mathrm{n}=349$ ) e sem sutura dos folhetos peritoneais (n = 349). Foram excluídas do estudo as mulheres com passado de laparotomia e/ou cesárea anterior, presença de febre ou quadro infeccioso (corioamnionite, infecção do trato urinário, infecção pulmonar, etc.), as com gestação com menos de 30 semanas e as que não receberam raquianestesia. A raquianestesia foi realizada no nivel de L3 e L4 com agulha ${ }^{\circ}$ 25-G descartável e a droga anestésica foi a bupivacaína hiperbárica na concentração de $0,5 \%$ associada a $0,2 \mathrm{mg}$ de sulfato de morfina. O estudo foi do tipo prospectivo, tipo ensaio clínico randomizado. Todas as parturientes foram submetidas à abertura da cavidade à Pfannenstiel, com histerotomia no segmento inferior, curvilinea, de concavidade cranial. A histerorrafia foi realizada em plano único com sutura contínua ancorada com catgute cromado (0). No grupo com fechamento do peritônio utilizou-se categute simples (00) em chuleio tanto para o visceral como o parietal. No grupo "não-fechamento", os peritônios visceral e parietal não foram suturados. Nos dois grupos houve a aproximação dos músculos retos com pontos separados e não-apertados, com fio de categute simples (00); sintese das aponeuroses, em plano singular, com pontos separados de categute cromado (0); sintese do subcutâneo com pontos separados de categute simples (00) e finalmente fechamento da pele com pontos separados de algodão (00).

Todos os procedimentos foram realizados por médicos plantonistas ou residentes (R2 ou R3) supervisionados por médicos preceptores. O tempo de cirurgia foi medido em minutos passados entre a abertura e o fechamento da pele. Também foi contado o número de fios categute simples utilizados na cesárea.

Não houve diferença estatisticamente significante entre os dois grupos em relação às variáveis demográficas (idade, paridade idade 
gestacional), uso de antibiótico profilático, indicações da cesárea, cefaléia pós-raquianestesia, cistite e amniorrexe prematura.

Quando a temperatura axilar foi maior ou igual a $38^{\circ} \mathrm{C}$, em pelo menos uma das medidas aferidas de 6 em 6 horas, no pós-operatório, foi considerada como febre. O uso de analgésico, óleo mineral, antifisético e antiemético foi aferido pela quantidade de doses administradas no pós-operatório e utilizados quando necessários. A presença de edema, calor, hiperemia e drenagem de secreção purulenta pela incisão, com ou sem febre, foi caracterizada como infecção da ferida operatória. O diagnóstico de endometrite foi dado quando presente febre, com útero subinvoluído, doloroso e amolecido à palpação, colo permeável, que manipulado deixa escoar secreção purulenta no pósoperatório. A permanência hospitalar no pós-operatório é apresentada em número de dias entre a cesárea e a alta hospitalar, considerando o dia da cesárea como dia zero.

A coleta dos dados foi realizada por meio do prontuário das mulheres. A digitação dos dados foi realizada, por pessoa única, sendo o banco de da- dos e a análise pelo Epi-Info 6.0. Foram utilizados quando apropriados os testes $t$ de Student e $\chi^{2}$ de Pearson com correção de Yates. Considerou-se a diferença estatisticamente significante quando o valor de $\mathrm{p}<0,05$ para todos os testes.

A pesquisa foi realizada após aprovação do Comitê de Ética em Pesquisa da Maternidade da Encruzilhada (CISAM/UPE).

\section{Resultados}

As variáveis tempo de cirurgia, número de unidades de fios utilizados e dias de internação foram maiores no grupo com peritonização quando comparadas com o grupo sem peritonização, e essa diferença foi estatisticamente significante. Não houve diferença estatisticamente significante entre os dois grupos em relação às variáveis febre, infecção da ferida operatória, endometrite, alta hospitalar e uso de antifisético, antiemético e óleo mineral (Tabela 1).

Tabela 1 - Resultados relacionados às variáveis cirúrgicas e do pós-operatório.

\begin{tabular}{|c|c|c|c|c|c|}
\hline & \multicolumn{2}{|c|}{$\begin{array}{l}\text { Com sutura peritoneal } \\
\qquad(\mathrm{n}=349)\end{array}$} & \multicolumn{2}{|c|}{$\begin{array}{l}\text { Sem sutura peritoneal } \\
\qquad(\mathrm{n}=349)\end{array}$} & $\mathrm{p}$ \\
\hline Tempo cirúrgico (minutos) & & & & & $<0,001$ \\
\hline $\mathrm{N}^{0}$ de fios (categute simples) & 3,93 & $(0,04)$ & 2,78 & $(0,26)$ & $<0,001$ \\
\hline Febre (n) & \multicolumn{2}{|c|}{29} & \multicolumn{2}{|c|}{26} & NS \\
\hline Infecção da FO (n) & \multicolumn{2}{|c|}{18} & \multicolumn{2}{|c|}{13} & NS \\
\hline Endometrite (n) & \multicolumn{2}{|c|}{5} & \multicolumn{2}{|c|}{5} & NS \\
\hline Uso de analgésico * & 3,5 & $(0,13)$ & 2,7 & $(0,12)$ & $<0,001$ \\
\hline Uso de antifisético & 1,0 & $(0,09)$ & 0,8 & $(0,08)$ & NS \\
\hline Uso de anti-emético & 0,05 & $(0,01)$ & 0,04 & $(0,01)$ & NS \\
\hline Uso de óleo mineral & 0,05 & $(0,02)$ & 0,03 & $(0,01)$ & NS \\
\hline Dias de internação & 3,7 & $(2,25)$ & 3,6 & $(2,33)$ & NS \\
\hline
\end{tabular}

FO - Ferida operatória

$\mathrm{n}=$ número de casos

${ }^{*} \mathrm{n}^{0}$ de doses

\section{Discussão}

Os dados mostram a equivalência dos dois grupos em confronto de variáveis demográficas (idade, paridade idade gestacional), uso de antibiótico profilático, cefaléia, cistite e amniorrexe prematura, deste modo evitando a interferência nos resultados a serem apreciados. Com a mesma finalidade de uniformização dos grupos em estudo, mostramos que as indicações da cesárea foram similares.

O tempo cirúrgico, o uso de analgésico e a quantidade de fios utilizados foram significativamente menores no grupo da não-peritonização do que no grupo em que a sintese peritoneal foi efetuada, resultados semelhantes aos encontrados por outros autores ${ }^{14,21}$. Não merece discussão o fato do menor número de fios gastos no primeiro grupo, por motivos mais do que óbvios. Igual afirma- 
ção pode ser feita no que diz respeito ao tempo cirúrgico, que foi expressivamente menor no grupo da não-peritonização, resultado este concordante com outros autores ${ }^{14,15,22}$.

A importância da diminuição do tempo operatório, quando não suturados os folhetos peritoneais na operação cesariana, pode se traduzir na diminuição das intercorrências febris ${ }^{18}$.

Outro achado que deve ser destacado é o uso de analgésicos, que foi menor no grupo sem sutura peritoneal do que naquele em que foi realizada a sintese do peritônio, resultado este também encontrado por outros autore ${ }^{15,22}$. A explicação do fenômeno reside na reação tipo corpo estranho acompanhada de inflamação que a sutura provoca nos folhetos peritoneais ${ }^{3,8,10,20}$.

Seria de imaginar-se que a técnica da operação cesariana realizada nos moldes atuais tivesse atingido o ápice da perfeição. A incisão de Pfannenstiel, para a abertura do ventre, na cesárea, preserva-lhe a estética. A histerotomia procura conservar a integridade da miofibrila e as suturas supostamente adequadas responsabilizavam-se por uma sintese quase integral. Outrossim, a antibioticoterapia e a anestesia progrediram no sentido do aperfeiçoamento, ajudando a transformar a operação cesariana em intervenção relativamente segura, apesar de percalços vários que ainda a povoam no que diz respeito à morbidade materna e, em menor grau, à mortalidade.

Publicações recentes vêm questionando a necessidade do fechamento do peritônio, tanto o visceral (prega vesicuterina) como o parietal, o que thes parece supérfluo, talvez inconveniente $\mathrm{t}^{14,15,16,18,19}$.

A proposta que ora se põe em discussão da não-sutura dos folhetos peritoneais, visceral e parietal, na verdade, não traduz em acréscimos técnicos ao ato operatório; ao revés disto, são subtraídos dois tempos operatórios da sintese que eram julgados imprescindiveis ao longo dos tempos. Se tais subtrações, realmente, traduzem-se em beneficio, é de se crer que a capacidade de recuperação do peritônio é de tal magnitude, que o fio de sutura, ao invés de beneficiá-la, serve-lhe de obstáculo, afirmativa essa respaldada por vários autores $^{1,3,4,5,7,8}$. Tais óbices, segundo Ellis ${ }^{1}$, seriam representados pela inflamação e pela isquemia determinados pelo fio de sutura, que em última análise redundariam num maior indice de aderências aos órgãos da vizinhança. Na investigação que ora realizamos não avaliamos ainda esta morbidade, dado o limite do tempo da pesquisa. Porém, será objetivo da continuidade do estudo a comparação de aderências entre os dois grupos quando da realização de eventual segunda cesárea.

\section{SUMMARY}

Purpose: to determine whether nonclosure of the visceral and parietal peritoneum is of benefit for the intraoperative or postoperative course of cesarean section.

Methods: six hundred and ninety-eight women scheduled for cesarean section were randomized to either closure of both visceral and parietal peritoneum $(n=349)$ or no peritoneal closure $(n=349)$, at the Maternidade da Encruzilhada (CISAM) in Recife, from November 1997 to December 1998. Statistical analysis compared intraoperative and postoperative outcome between the two groups. There was no difference regarding age, parity, gestational age, antibiotic prophylaxis, headache after spinal anesthesia, cystitis, ruptured membranes and indications for cesarean section.

Results: operating time, number of absorbable sutures and use of analgesics were significantly lower in the group without closure. The incidence of febrile morbidity, wound infection and endometritis was similar in the two groups. There was no difference in the need for antiphysetics, antiemetics and mineral oil. The average hospital stay was similar in the two groups.

Conclusions: nonclosure of the visceral and parietal peritoneum at cesarean section was not associated with adverse effects on the postoperative course; on the contrary, it reduced the use of analgesics, and intraoperatively it reduced operating time and the number of absorbable sutures.

KEY WORDS: Cesarean section. Surgery: complications. Puerperal infection.

\section{Referências}

1. Ellis H. The cause and prevention of postoperative intraperitoneal adhesions. Surg Gynecol Obstet $1971 ; 133: 497-511$.

2. Weibel MA, Majno G. Peritoneal adhesions and their relation to abdominal surgery. Am J Surg 1973; 126:345-53.

3. Ellis H. The etiology of postoperative abdominal adhesions. Br J Surg 1962; 50:10-17.

4. Ellis H, Harrison W, Hugh TB. The healing of peritoneum under normal and pathological conditions. Br J Surg 1965; 52:471-6.

5. Conolly WB, Stephens FO. Factors influencing intraperitoneal adhesions: an experimental study. Surgery 1968; 63:976-9. 
6. Buckman RFJr, Buckman PD, Hufnagel HV, Gervin AS. A physiologic basis for the adhesion-free healing of de-peritonealized surfaces. J Surg Res 1976; 21:67-76.

7. Kapur BML, Daneswar A, Chopra P. Evaluation of peritoneal closure at laparotomy. Am J Surg 1979; 137:650-2.

8. Elkins TE, Stovall TG, Warren J, Ling FW, Meyer NL. A histologic evaluation of peritoneal injury and repair: implications for adhesion formation. Obstet Gynecol 1987; 70:225-8.

9. O'Leary DP, Coakley JB. The influence of suturing and sepsis on the development of postoperative peritoneal adhesions. Ann R Coll Surg Engl 1992; 74:134-7.

10.Ellis H, Heddle R. Does the peritoneum need to be closed at laparotomy? Br J Surg 1977; 64:733-6.

11.Spernol R, Hecher K, Gorzer H, Szalay S. Complications of radical operation of uterine cancer. Closure of peritoneal defects - yes or no? Geburtshilfe Frauenheilkd 1992; 52:210-3.

12.Gilbert JM, Ellis H, Foweraker S. Peritoneal closure after lateral paramedian incision. Br J Surg 1987; 74:113-5.

13.Tulandi T, Hum HS, Gelfand MM. Closure of laparotomy incisions with ou without peritoneal suturing and second-look laparoscopy. Am J Obstet Gynecol 1988; 158:536-7.

14.Pietrantoni M, Parsons MT, O’Brien WF, Collins E, Knuppel RA, Spellacy WN. Peritoneal closure or non-closure at cesarean. Obstet Gynecol 1991; 77:293-6.
15. Hull DB, Varner MW. A randomized study of closure of the peritoneum at cesarean delivery. Obstet Gynecol 1991; 77:818-21.

16.Stark M, Chavkin Y, Kupfersztain C, Guedj P, Finkel AR. Evaluation of combinations of procedures in cesarean section. Int J Gynaecol Obstet 1995; 48:273-6.

17. Ohel G, Younis JS, Lang N, Levit A. Doublelayer closure of uterine incision with visceral and parietal peritoneal closure: are they obligatory steps of routine cesarean sections? J Matern Fetal Med 1996; 5:3669.

18.Nägele F, Karas H, Spitzer D, Staudach A, Karasegh $\mathrm{S}$, Beck A, et al. Closure or nonclosure of the visceral peritoneum at cesarean delivery. Am J Obstet Gynecol 1996; 174:1366-70.

19.Irion O, Luzuy F, Beguin F. Non-closure of the visceral and parietal peritoneum at caesarean section: a randomized controlled trial. $\mathrm{Br} \mathrm{J}$ Obstet Gynaecol 1996; 103:690-4.

20.Wadstrom J, Gerdin B. Closure of the abdominal wall: How and Why? Acta Chir Scand 1990; 156:7582.

21.Grundsell HS, Rizk DE, Kumar RM. Randomized study of non-closure of peritoneum in lower segment cesarean section. Acta Obstet Gynecol Scand 1998; 77:110-15.

22.Darj E, Nordström M-L. The Misgav Ladach method for cesarean section compared to the Pfannenstiel method. Acta Obstet Gynecol Scand $1999 ; 78: 37-41$. 